\title{
SOME RESULTS ON THE REAL $K$-THEORY OF CERTAIN HOMOGENEOUS SPACES
}

\author{
M.O. A JETUNMOBI
}

Abstract

Let $F_{r}(n)$ be the incomplete complex flag manifold of length $r$ in $\mathbb{C}^{n}$. We make a start on the complete determination of the torsion part of the group $K O^{-i}\left(F_{r}(n)\right)$ giving results here when $r=2,3$.

Introduction and Statement of results. Let $K O(X)$ be the Grothendieck ring of real vector bundles over a space $X$, and $\mathrm{KO}^{-i}$, the associated cohomology theory. Let $F_{r}(n)$ be the incomplete complex flag manifold of length $r$ in $\mathbb{C}^{n}$ which can be identified with

$$
\frac{U(n)}{U(1)^{r} \times U(n-r)}
$$

where $U(1)^{r}=U(1) \times U(1) \times \cdots \times U(1)(r$ factors $)$.

The question raised is "how many copies of $Z_{2}$ are present in $\mathrm{KO}^{-i}\left(F_{r}(n)\right)$ ". A similar question was asked by Hoggar in [3] for the complex Grassmannian. The free part of $\mathrm{KO}^{-i}\left(F_{r}(n)\right)$ is completely known from $[3]$. In this paper, we make a start on the determination of the torsion part of $\mathrm{KO}^{-i}\left(F_{r}(n)\right)$ giving results here when $r=2,3$. The case $r=1$ is done in [2].

Proposition A. The rank of $K O^{-2 i}\left(F_{r}(n)\right)$ is $\frac{n !}{2(n-r) !}, i=0,1,2,3$.

Theorem A. The $\mathrm{KO}^{-i}$-groups of the complex flag manifold of length two, $F_{2}(n)$ are as follows:

\begin{tabular}{|c|c|c|}
\hline$\underline{i}$ & $n \equiv 0,1,3(\bmod 4)$ & $n \equiv 2(\bmod 4)$ \\
\hline 0 & 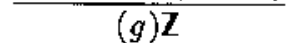 & $(g) \mathbf{Z} \oplus \mathbf{Z}_{2}$ \\
\hline 1 & $\mathrm{Z}_{2}$ & $Z_{2}$ \\
\hline 2 & $(g) \mathbf{Z} \oplus \mathbf{Z}_{2}$ & $(g) \mathbf{Z} \oplus \mathbf{Z}_{\mathbf{2}}$ \\
\hline 3 & $\mathbf{Z}_{2}$ & 0 \\
\hline 4 & $(g) \mathbf{Z} \oplus \mathbf{Z}_{2}$ & $(g) \mathbf{Z}$ \\
\hline 5 & 0 & 0 \\
\hline 6 & (g) $\mathbf{Z}$ & (g) $\mathbf{Z}$ \\
\hline$f$ & 0 & $\mathbf{Z}_{2}$ \\
\hline
\end{tabular}


where $g=\frac{n(n-1)}{2}$ and $(g) \mathbb{Z}$ means the free abelian group of rank $g$.

Theorem B. The $\mathrm{KO}^{-i}$-groups of $F_{3}(n)$ are as follows:

$\begin{array}{cccc}\underline{i} & \frac{n o d d}{(\ell) \mathbf{Z}} & \frac{n \equiv 0(\bmod 4)}{(\ell) \mathbf{Z}} & \frac{n \equiv 2(\bmod 4)}{(\ell) \mathbf{Z} \oplus \mathbf{Z}_{2}} \\ 0 & \mathbf{Z}_{2} & \mathbf{Z}_{2} & \mathbf{Z}_{2} \oplus \mathbf{Z}_{2} \\ 1 & (\ell) \mathbf{Z} \oplus \mathbf{Z}_{2} & (\ell) \mathbf{Z} \oplus \mathbf{Z}_{2} & (\ell) \mathbf{Z} \oplus \mathbf{Z}_{2} \oplus \mathbf{Z}_{2} \\ 2 & \mathbf{Z}_{2} & \mathbf{Z}_{2} \oplus \mathbf{Z}_{2} & \mathbf{Z}_{2} \\ 3 & (\ell) \mathbf{Z} \oplus \mathbf{Z}_{2} & (\ell) \mathbf{Z} \oplus \mathbf{Z}_{2} \oplus \mathbf{Z}_{2} & (\ell) \mathbf{Z} \oplus \mathbf{Z}_{2} \\ 4 & 0 & \mathbf{Z}_{2} & 0 \\ 5 & (\ell) \mathbf{Z} & (\ell) \mathbf{Z} \oplus \mathbf{Z}_{2} & (\ell) \mathbf{Z} \\ 6 & 0 & 0 & \mathbf{Z}_{2} \\ 7 & & & \end{array}$

where $\ell=\frac{n(n-1)(n-2)}{2}$.

1. Proof of Proposition A. We observe that since the rational $K O$ Atiyah-Hirzebruch spectral sequence [1] for $F_{r}(n)$ collapses, we have

$$
\operatorname{rank} K O^{\circ}\left(F_{r}(n)\right)+\operatorname{rank} K O^{-2}\left(F_{r}(n)\right)=\operatorname{rank} H^{e v}\left(F_{r}(n)\right)=\frac{n !}{(n-r) !}
$$

and the result follows from the fact (see [3]) that rank $K O^{2 i}\left(F_{r}(n)\right)=\operatorname{rank}$ $K O^{2 i+2}\left(F_{r}(n)\right), i=0,1$.

2. Proof of Theorem A. First we give generators of $H^{*}\left(F_{2}(n) ; K O^{*}\left(\left\{{ }^{*}\right\}\right)\right)$, where $\{*\}$ denotes a point space.

There are two canonical complex line bundles $h_{1}, h_{2}$ over $F_{2}(n)$.

Put $x_{1}=c_{1}\left(h_{1}\right), y_{1}=c_{1}\left(h_{2}\right)$, where $c_{1}$ is the first Chern class. Let $x, y$ be the mod 2 reductions of $x_{1}, y_{1}$ respectively, and $H^{*}\left(F_{2}(n) ; K O^{*}\left({ }^{*}\right)\right)$ is generated as a $K O^{*}\left({ }^{*}\right)$ algebra by either $x, y$ or by $x_{1}, y_{1}$. In fact, an additive basis for $H^{*}\left(F_{2}(n) ; K O^{*}\left({ }^{*}\right)\right)$ is $x^{i} y^{j}$ or $x_{1}^{i} y_{1}^{j}(0 \leq i \leq n-1 ; 0 \leq j \leq n-2)$ and the ring structure is given by $H^{*}\left(F_{2}(n) ; Z\right]=\mathbb{Z}\left[x_{1}, y_{1}\right]$ subject to

$$
\bar{\sigma}_{n-1}\left(x_{1}, y_{1}\right)=0=\bar{\sigma}_{n}\left(x_{1}, y_{1}\right)
$$

and similar result holds for $H^{*}\left(F_{2}(n) ; \mathbf{Z}_{2}\right)$ where $\bar{\sigma}_{i}$ is the $i^{\text {th }}$ complete symmetric function in $x_{1}$ and $y_{1}$, that is, the sum of all monomials of degree $i$ in $x_{1}$ and $y_{1}$, and it is to be interpreted as 1 when $i=0$ and as zero when $i<0$.

$\operatorname{Kerd}_{2}^{p, q}$ In the $\mathrm{KO}$-theory Atiyah-Hirzebruch spectral sequence [1], we consider the differential

$$
E_{2}^{p, q} \stackrel{d_{2}^{p, q}}{\rightarrow} E_{2}^{p+2, q-r}
$$

Then using lemma (2.4) in [3],

$$
d_{2}^{p, q}=0 \text { for } q \equiv-2,-4(\bmod 4) \text {. }
$$


$d_{2}^{p_{+}-8 t}=S q^{2} \rho_{2}$ and $d_{2}^{p,-8 t-1}=S q^{2}$. For properties of $S q^{2}$ see [4].

Now, using the Cartan formula,

$$
S q^{2}\left(x^{r} y^{s}\right)=\left\{\begin{array}{l}
0: s, r \text { even } \\
x^{r} y^{s+1}+x^{r+1} y^{s}: r, s \text { odd }
\end{array}\right.
$$

for $r+s \equiv 0,2(\bmod 4)$. Also, for $r+s \equiv 1,3(\bmod 4)$

$$
S q^{2}\left(x^{r} y^{s}\right)=\left\{\begin{array}{c}
x^{r+1} y^{s}: r \text { odd, } s \text { even } \\
x^{r} y^{s+1}: s \text { odd, } r \text { even } .
\end{array}\right.
$$

Hence, for $n$ even and in dimensions $\leq 2(n-2)$ (writing down the number of copies of the coefficient group, and $\beta_{i}$ represents the $i^{\text {th }}$ Betti number)

$$
\begin{gathered}
\operatorname{Ker} d_{2}^{8 k, q}=\left[\frac{1+\beta_{8 k}}{2}\right], \operatorname{Im} d_{2}^{8 k, q}=\left[\frac{\beta_{8 k}}{2}\right] \\
\operatorname{Ker} d_{2}^{8 k, q}=\left[\frac{\beta_{8 k}}{2}\right], \operatorname{Im} d_{2}^{8 k+2, q}=\left[\frac{1+\beta_{8 k+4}}{2}\right] .
\end{gathered}
$$

We obtain the same values for $n$ odd except

$$
\begin{gathered}
\operatorname{Ker}_{2}^{2(n-1), q}=\left[\frac{\beta_{2(n-1)}}{2}\right]+1=\frac{n+1}{2}, \operatorname{Im} d_{2}^{2(n-1), q}=\left[\frac{\beta_{2(n-1)}}{2}\right]-1=\frac{n-3}{2} . \\
\operatorname{Ker} d_{2}^{2(n-2), q}=\left[\frac{\beta_{2(n-2)}}{2}\right]-1, \operatorname{Im} d_{2}^{2(n-2), q}=\left[\frac{\beta_{2(n-1)}}{2}\right]+1=\frac{n+1}{2} .
\end{gathered}
$$

For dimensions $\geq 2(n-1)$

$n$ even

$$
\begin{gathered}
\operatorname{Ker} d_{2}^{8 k, q}=\left[\frac{\beta_{8 k}}{2}\right], \operatorname{Im} d_{2}^{8 k, q}=\left[\frac{\beta_{8 k+2}}{2}\right]+1 \\
\operatorname{Ker}_{2}^{8 k+2, q}=\left[\frac{\beta_{8 k+2}}{2}\right]+1, \operatorname{Im} d_{2}^{8 k+2, q}=\left[\frac{\beta_{8 k+4}}{2}\right]
\end{gathered}
$$

$n$ odd

$$
\begin{aligned}
\operatorname{Ker} d_{2}^{8 k, q} & =\left[\frac{\beta_{8 k}}{2}\right]+1, \operatorname{Im} d_{2}^{8 k, q}=\left[\frac{\beta_{8 k+2}}{2}\right] \\
\operatorname{Ker} d_{2}^{8 k+2, q} & =\left[\frac{\beta_{8 k+2}}{2}\right], \operatorname{Im} d_{2}^{8 k+2, q}=\left[\frac{\beta_{8 k+4}}{2}\right]+1
\end{aligned}
$$

for $q \equiv 0,-1(\bmod 8)$.

Remark. Similar results hold for

$$
\operatorname{Ker} d_{2}^{8 k+4, q} \text { and } \operatorname{Ker} d_{2}^{8 k+6, q} \text {. }
$$


Torsion Part:

Now consider the sequence of differentials

$$
E_{2}^{p-2, q+1} \rightarrow E_{2}^{p, q} \rightarrow E_{2}^{p+2, g-1} .
$$

For $q \equiv-1(\bmod 8),(1)$ becomes

$$
E_{2}^{p-2,-8 t} \rightarrow E_{2}^{p,-8 t-1} \rightarrow E_{2}^{p+2,-8 t-2} .
$$

For $p \equiv 0(\bmod 8)$, we get

$$
E_{3}^{8 k_{1}-8 t-1}=0, k \neq 0 \text { and } E_{3}^{0_{1}-8 t-1}=\mathbf{Z}_{2} .
$$

Similarly for $p \equiv 2(\bmod 8)$,

$$
\begin{gathered}
E_{3}^{8 k+2,-8 t-1}=0 \text { for } 8 k+2 \neq 2(n-1), n \equiv 2(\bmod 4) \\
E_{3}^{2(n-1),-8 t-1}=Z_{2}(n \equiv 2(\bmod 4)) .
\end{gathered}
$$

Also, for $p \equiv 4(\bmod 8)$

$$
E_{3}^{8 k+4,-8 t-1}=0 .
$$

Finally for $p \equiv 6(\bmod 8), E_{3}^{8 k+6_{1}-8 t-1}=0$ for $8 k+6 \neq 2(n-1)(n$ even $)$ or $8 k+$ $6 \neq 4 n-6(n$ odd $), E_{3}^{2(n-1),-8 i-1}=\mathbb{Z}_{2}(n \equiv 0(\bmod 4))$, and $E_{3}^{4 n-6,-8 t-1}=\mathbb{Z}_{2}$ $(n$ odd).

For $q \equiv-2(\bmod 8)$, from $(1)$ we get

$$
E_{3}^{p,-8 t-2}=\frac{E_{2}^{p,-8 t-2}}{\operatorname{Im} d_{2}^{p-2,-8 t-1}} .
$$

Now we show that $E_{3}=E_{\infty}$ for $q \equiv-1(\bmod 8)$.

From the differential $E_{r}^{0,-1} \stackrel{d_{r}}{\rightarrow} E_{r}^{r_{r}-r}, E_{r}^{r_{1}-r}=0$ except $r \equiv 0,2,4(\bmod 8)$ and $d_{r}=0$ for $r \equiv 0,4(\bmod 8)$ because it maps a finite group to a free group. Thus, we are left with the case $r \equiv 2(\bmod 8) . E_{3}^{0,-1}$ is generated by unity, 1 , and we claim that $d_{r}(1)=0$ for $r \equiv 2(\bmod 8)$.

Proof of Claim: It suffices to show that $d_{10}(1)=0$. The differential $d_{r}$ is a derivation which implies (by induction) that $d_{10}\left(x^{s}\right)=s x^{s-1} d_{10}(x)$ and the claim follows. Hence $E_{3}^{0,-1}=E_{\infty}^{0,-1}$. By considering the filtration of $K O^{-1}$, we have $K O^{-1}=Z_{2}$ for all $n$.

For $n$ even, we consider the sequence of differentials

$$
E_{r}^{2(n-1)-r, r-2} \stackrel{d_{r}}{\rightarrow} E_{r}^{2(n-1),-1} \stackrel{d_{r}}{\rightarrow} E_{r}^{2(n-1)+r_{r}-r}
$$

$E_{\tau}^{2(n-1)-r, r-2}=0$ except for $r \equiv 0,2,6(\bmod 8),{ }^{\prime} d_{r}=0$ for $r \equiv 0,6(\bmod 8)$ (see $[3])$ and $' d_{r}=0$ for $r \equiv 2(\bmod 8)$ because $E_{r}^{2(n-1)-r, 0}$ is a free group and 
it survives to $E_{\infty}$. Thus ' $d_{r}=0$ for all $r \geq 3$. Also $E_{r}^{2(n-1)+r,-r}=0$ except for $r \equiv 0,2,4(\bmod 8)$ in which case $" d_{r}=0$ for $r \equiv 0,4(\bmod 8)$ because it maps a finite group to a free group. Thus we are left with the differential

$$
E_{8 k+2}^{2(n-1),-1} \stackrel{. " d_{g k+2}}{\rightarrow} E_{8 k+2}^{2 n+8 k,-2} .
$$

We claim that $" d_{8 k+2}=0$.

Proof of Claim: It is clear that $E_{3}^{2(n-1),-1}=Z_{2}$ is generated by $x^{n-1}$. We recall that $x$ and $y$ are independent and any cohomology operation $\theta\left(x^{r}\right)$ is a function of $x$ only while $\theta\left(y^{*}\right)$ is a function of $y$ only for positive integers $r, s$, and

$$
" d_{8 k+2}\left(x^{n-1}\right)=(n-1) x^{n-2} d_{8 k+2}(x)=x^{n-2} d_{8 k+2}(x) .
$$

Since $d_{8 k+2}(x)$ is a function of $x$ only and $x^{n-2} d_{8 k+2}(x) \varepsilon H^{2 n+8 k}\left(F_{2}(n) ; \mathrm{Z}_{2}\right)$ and using the constraint $x^{n}=0$ we have $d_{8 k+2}=0$ finishing the proof of claim. Hence $E_{3}^{2(n-1),-1}$ survives to $E_{\infty}$. Thus by considering the filtration of $K O^{2 n-3}$, we have $K O^{2 n-3}=\mathrm{Z}_{2}, n$ even $(\bmod 4)$ i.e.

$$
K O^{2 n-3}=\left\{\begin{array}{l}
K O^{-3} n \equiv 0(\bmod 4) \\
K O^{-7} n \equiv 2(\bmod 4) .
\end{array}\right.
$$

For $n$ odd, we consider the differential $E_{r}^{4 n-6-r, r-2} \stackrel{d_{r}}{\rightarrow} E_{r}^{4 n-6,-1}$, and by similar reasoning, $E_{3}^{4 n-6,-1}=E_{\infty}^{4 n-6,-1}$. By considering the filtration of $K O^{4 n-7}$, we have $K O^{4 n-7}=K O^{-3}=Z_{2}$.

The free parts follow from proposition (A). These and the torsion part complete the proof of Theorem (A).

3. Conclusion. The proof of theorem (B) is exactly similar to that in 2 but the procedure for determining the kernels and the images of the differentials is fairly elaborate.

Remark: By using the double structure of $F_{r}(n)$ as the total space of a bundle over the Grassmannian and as the total space of a projective bundle over $F_{r-1}(n)$, we can apply the $K O$-spectral sequence of a fibration to the spherical fibration $\pi: F_{2}(n) \rightarrow G_{2}\left(C^{n}\right)$ with fibre $S^{2}$, the 2-sphere and the projective fibration $\alpha: F_{2}(n) \rightarrow \mathbf{P}^{n-1}(\mathrm{C})$ with fibre $P^{n-2}(\mathcal{C})$ to obtain $K O^{-i}\left(F_{2}(n)\right)$.

In particular, for $n$ odd, theorem $A$ agrees with the results of Karoubi in [5].

\section{References}

1. M.R. AtiYah, F. HiRzebruch, Vector bundles and homogeneous spaces, Proc. of Symposia in Pure Math., Differential Geometry 3 (1961), 7-36, Amer. Math. Soc. 
2. M. FUIII, KO-groups of projective spaces, Osaka Jour. Math. 4 (1967), 141-149.

3. S.G. HoGGAR, On KO-theory of Grassmannian, Quart. Jour. Math, Oxford Ser. (2) 20 (1969), 477-463.

4. D. HUSEMOLLER, Fibre bundles, Springer-Verlag (1975), New York. Heidelberg Berlin - Second edition.

5. M. Karoubi, V. MUdRINSKI, Real $K$-theory of Complex Projective Bundles, Acad. Sc. Paris 297 Series 1 (1983), 349-352.

\author{
Facuity of Science \\ Lagos State University \\ Ojo, Badegry expressway \\ Lagos, NIGERIA.
}

Rebut el 10 de Novembre de 1987 